\title{
La Eficacia del Paradigma de Producción para Promover la Recuperación de Análogos Interdominio
}

\section{The Effectiveness of the Production Paradigm in Eliciting Cross-Domain Analogical Retrieval}

\author{
Máximo Trench y Nicolás Oberholzer \\ Universidad de Buenos Aires
}

\author{
Fernando Adrover \\ Universidad Abierta Interamericana
}

\author{
Ricardo Minervino \\ Universidad Nacional del Comahue
}

\begin{abstract}
Los estudios de recuperación analógica que emplean un paradigma de recepción muestran que la recuperación interdominio es menor que la recuperación intradominio. Utilizando un paradigma de producción, Blanchette y Dunbar (2000) encontraron resultados opuestos. En un primer experimento replicamos este estudio, utilizando materiales que le confieren mayor validez. En un segundo experimento pusimos a prueba la hipótesis que, cuando los análogos base y meta reciben una codificación estructural, se generan los altos niveles de recuperación interdominio observados bajo un paradigma de producción. Nuestros resultados mostraron que la recuperación interdominio resultó escasa y menos frecuente que la recuperación intradominio. La explicación dada por Blanchette y Dunbar acerca de la eficacia del paradigma de producción para promover recuperaciones interdominio parece insuficiente.
\end{abstract}

Palabras clave: analogía, recuperación, transferencia, memoria

\begin{abstract}
Experimental studies using a reception paradigm show that interdomain analog retrieval is intrinsically difficult, and far less likely to occur than intradomain analogue retrieval. Using a production paradigm, Blanchette and Dunbar (2000) obtained contradictory results. In Experiment 1 we replicated Blanchette and Dunbar's study using materials that give more validity to their results. Experiment 2 was designed to test the hypothesis that a structural encoding of the base and target analogs promotes the high rate of interdomain retrieval observed under a production paradigm. Our results showed that interdomain retrieval was scarce and less frequent than intradomain retrieval, suggesting that the hypothesis advanced by Blanchette and Dunbar doesn't account for the data obtained using a production paradigm.
\end{abstract}

Keywords: analogy, retrieval, transfer, memory.

Introducción

El pensamiento a través de analogías supone la transferencia de conocimiento desde una situación conocida (análogo base: $\mathrm{AB}$ ) hacia una situación novedosa (análogo meta: AM) cuya comprensión se busca mejorar, y forma parte de variadas activi- dades cognitivas, como la solución de problemas, el descubrimiento científico o la argumentación (Gentner, Holyoak \& Kokinov, 2001; Minervino \& Oberholzer, 2007).

Dos situaciones son análogas si sus entidades están organizadas por sistemas de relaciones similares (Gentner, 1983; Holyoak \& Thagard, 1995),

Máximo Trench, Universidad de Buenos Aires, Consejo Nacional de Investigaciones Científicas y Técnicas, Buenos Aires, Argentina.

Nicolás Oberholzer, Universidad de Buenos Aires, Argentina.

J. Fernando Adrover, Universidad Abierta Interamericana, Buenos Aires, Argentina.

Ricardo A. Minervino, Universidad Nacional del Comahue, Consejo Nacional de Investigaciones Científicas y Técnicas, Neuquén, Argentina.

La correspondencia relativa a este artículo debe ser dirigida a Juan Máximo Trench, Av. Figueroa Alcorta 3060 11B, 1425, Ciudad Autónoma de Buenos Aires, Argentina. E-mail: mtrench@psi.uba.ar

Este trabajo ha sido posible gracias al apoyo de los subsidios UBACYT P030, concedido por la Universidad de Buenos Aires, y PICT33150, concedido por la Agencia Nacional de Promoción Científica y Tecnológica de Argentina al cuarto autor de este trabajo. 
esto es, si guardan una semejanza estructural. En una analogía interdominio (e.g., el símil entre el átomo y el sistema solar) las situaciones comparadas pertenecen a ámbitos semánticos distantes, por lo que los objetos que se ponen en correspondencia (e.g., el sol y el núcleo del átomo) no poseen semejanzas. En una analogía intradominio (e.g., la comparación entre la Crisis del Golfo y la Segunda Guerra Mundial) las situaciones comparadas provienen de un mismo dominio temático, por lo que los objetos emparejados tienden a ser intrínsecamente similares, esto es, instancias de una misma categoría supraordenada inmediata (e.g., Alemania e Iraq son dos casos de país). Al parecido entre los objetos de dos análogos se lo llama semejanza superficial (Gentner, 1989; Holyoak \& Thagard, 1995). De esta forma, las analogías intradominio guardan, además de una semejanza estructural, una semejanza superficial.

Numerosos estudios han mostrado que la recuperación de $\mathrm{AB}$ desde nuestra memoria de largo plazo (MLP) solo resulta fácil cuando se trata de analogías intradominio, siendo las recuperaciones interdominio relativamente infrecuentes (Catrambone, 2002; Gentner, Rattermann \& Forbus, 1993; Rattermann \& Gentner, 1987; Wharton, Holyoak, Downing, Lange, Wickens \& Melz, 1994; Wharton, Holyoak \& Lange, 1996). Estos resultados se han obtenido empleando un paradigma que, al igual que en la mayoría de los estudios sobre memoria, consta de una fase de codificación y una fase de recuerdo. Mientras que en la fase de codificación se les presenta a los participantes un conjunto de $\mathrm{AB}$, en la fase de recuperación se les presenta una serie de $\mathrm{AM}$ y se les pregunta a qué $\mathrm{AB}$ de la fase anterior estos AM les hacen recordar. Empleando este tipo de procedimiento -al que Blanchette y Dunbar (2000) denominaron paradigma de recepción-, Gentner et al. (1993) obtuvieron una tasa de recuperación de $\mathrm{AB}$ intradominio del $59 \%$, en tanto que la tasa de recuperación de $\mathrm{AB}$ interdominio resultó en un $21 \%$. Los resultados de los estudios nombrados y otros similares (para una revisión véase De la Fuente \& Minervino, 2004) muestran que la tasa de recuperaciones intradominio es entre dos y tres veces más elevada que la tasa de recuperaciones interdominio, y que el nivel de recuperación de $\mathrm{AB}$ interdominio raramente supera el $20 \%$, lo que sugiere que las semejanzas superficiales constituyen una condición de mucho peso para la recuperación.

Empleando un procedimiento alternativo al paradigma de recepción, Blanchette y Dunbar (2000, Experimento 2) obtuvieron resultados inconsis- tentes con los de los estudios tradicionales. Este procedimiento -llamado por los autores paradigma de producción- consiste en presentar una situación meta y pedir a los participantes que generen analogías para esa situación, sin que el experimentador haya previamente presentado $\mathrm{AB}$ que les podría ser de utilidad. El AM usado por estos autores trataba sobre el tema del creciente déficit fiscal canadiense, y la tarea meta consistió en generar analogías persuasivas a favor (o en contra, según la condición) de adoptar una política de déficit cero. Tras conceder 20 minutos para la realización de esta tarea, obtuvieron una elevada producción general de analogías (10,73 analogías por persona), con un marcado predominio de analogías interdominio por sobre las analogías intradominio ( $80 \%$ vs. 20\%). Blanchette y Dunbar (2000) y Dunbar (2001) consideraron que este predominio de analogías interdominio podría deberse al efecto combinado de dos factores: 1) la tarea de generación de analogías -a diferencia de la tarea de recuerdo típica del paradigma de recepción- favorece la extracción de claves estructurales del AM, las que permitirían la recuperación de $\mathrm{AB}$ que no mantienen similitudes superficiales con el AM, y 2) la codificación natural de los $A B$-a diferencia de la codificación propia de los contextos experimentales, que estimulan un procesamiento superficial (Blanchette \& Dunbar, 2000)- destaca aspectos estructurales de los mismos. Las codificaciones estructurales tanto del $\mathrm{AB}$ como del AM propias del paradigma de producción -y presentes en menor medida en el paradigma de recepción- explicarían por qué este paradigma da lugar a un predominio de analogías interdominio.

Blanchette y Dunbar (2000) consideraron que una objeción contra la validez de los resultados obtenidos en su Experimento 2 podría consistir en que, dado que el tema meta del déficit cero había recibido una gran cobertura mediática en los tiempos previos al estudio, los participantes podrían haber recuperado analogías previamente establecidas por otras personas en vez de haber generado las suyas propias. Si así fuese, sus datos no serían contradictorios con los datos procedentes del paradigma de recepción, ya que en estos se evalúa recuperación de $\mathrm{AB}$ y no recuperación de analogías desde la MLP.

El primero de los dos experimentos del presente estudio tuvo como propósito replicar el Experimento 2 de Blanchette y Dunbar (2000), utilizando materiales que no permitieran poner en duda la validez de sus resultados por la razón descrita, esto es, empleando materiales para los que las probabilidades de 
recuperar analogías preexistentes fueran menores. El segundo experimento tuvo como propósito poner a prueba la hipótesis de Blanchette y Dunbar (2000) y Dunbar (2001) relativa a que la combinación de AB codificados estructuralmente y una tarea meta de generación de analogías resultan en altas tasas absolutas y relativas de recuperación de $\mathrm{AB}$ interdominio.

\section{Experimento 1}

Como hemos dicho, Blanchette y Dunbar (2000) consideraron que una posible objeción a la validez de los resultados obtenidos en su Experimento 2 podría consistir en que los participantes recuperaron analogías de las que ya disponían, en vez de generar analogías propias. Los autores juzgaron que esta objeción es atendible ya que el debate sobre el déficit cero había recibido una enorme cobertura mediática en los meses previos a la realización del estudio. Si a esta objeción se le suma el hecho que estudios anteriores (e.g., Blanchette \& Dunbar, 1997) demostraron que las analogías políticas utilizadas en los medios resultan predominantemente interdominio, los resultados obtenidos por Blanchette y Dunbar podrían ser explicados de este modo: el predominio de analogías interdominio en el paradigma de producción se debe a que se recuperan analogías ya conocidas para el AM y a que estas analogías tienden a ser interdominio.

En nuestro primer experimento nos propusimos replicar los hallazgos de Blanchette y Dunbar (2000, Experimento 2) empleando materiales para los que fuera menos probable que los participantes recuperasen analogías ya conocidas en vez de generar nuevas analogías. Con este propósito decidimos utilizar diversos AM que tratasen sobre temas que, de acuerdo a nuestra intuición, no fueran de amplia discusión pública ni hubieran recibido tratamiento en los medios. Dado que no es sencillo determinar el grado en que una analogía es individual y novedosa o es una variación de una analogía convencional culturalmente compartida (Lakoff \& Turner, 1997; Minervino, 2007), decidimos presentar las analogías propuestas por los participantes a un grupo independiente, que tuvo que juzgar cuán novedosa le parecía cada analogía. El propósito de este control fue probar si, tal como lo suponíamos, nuestros temas meta evitaban dar lugar al reporte de analogías preexistentes culturalmente compartidas para el tema meta. Con el objetivo de aumentar el grado de generalización de los resultados obtenidos, en este primer experimento se incluyeron $10 \mathrm{AM}$ diferentes, a diferencia de Blanchette y Dunbar (2000) que trabajaron solo con uno.

\section{Método}

Participantes. Treinta estudiantes de segundo año de Psicología de la Universidad de Buenos Aires tomaron parte voluntariamente en el experimento. La muestra estuvo constituida por 23 mujeres y 7 varones, con edades entre 18 y 41 años $(M=21,67$, $D E=4,71)$.

Procedimiento y materiales. Aunque el experimento fue administrado en pequeños grupos, los participantes trabajaron de manera individual. Se les comunicó que participarían en dos ejercicios de argumentación mediante analogías, en las que deberían persuadir a otras personas acerca de la conveniencia de adoptar una línea de acción predeterminada (Blanchette \& Dunbar, 2000, Experimentos 1 y 2 , no encontraron diferencias entre la tarea de argumentar a favor o en contra de una idea, por lo cual esta variable no fue manipulada). Las consignas y tareas fueron presentadas por escrito en un cuadernillo de ocho hojas, en cuyas dos primeras se explicaba en qué consiste hacer una analogía para persuadir y se les proporcionaban dos ejemplos (una analogía intradominio y otra interdominio). En las siguientes hojas se presentaban los dos AM, cada uno en una hoja aparte, seguidos de dos hojas en blanco para escribir analogías. Los participantes trabajaron durante 15 minutos con cada tarea meta. Tanto las tareas meta elegidas para cada participante como su orden de presentación fueron contrabalanceados. Se utilizaron como AM 10 situaciones con final abierto, cuya extensión variaba entre 220 y 240 palabras. En todas las historias alguno de los personajes debía optar entre dos posibles líneas de acción. Por ejemplo, en una de ellas un investigador se debatía con respecto a si convenía destruir las últimas muestras existentes del virus de la viruela, considerando, por un lado, su valor para la investigación $\mathrm{y}$, por otro, el riesgo potencial de que pudieran originar nuevos casos de la enfermedad. Los participantes debían escribir todas las analogías que pudieran para convencerlo de la conveniencia de destruir las últimas muestras existentes (para más ejemplos de los materiales empleados, véase el Anexo 1).

Análisis de datos. Dos jueces recibieron instrucción acerca del concepto de analogía y de la distinción entre analogías intra e interdominio. Cada una de las propuestas de analogías dadas por los partici- 
pantes fue analizada independientemente por los dos jueces. El acuerdo entre jueces fue del $90 \%$ respecto al carácter analógico o no analógico de las propuestas (Kappa $=0,49, p<0,001)$ y del $78 \%$ con respecto al carácter intra o interdominio de las analogías (Kappa $=0,47, p<0,001)$. Los desacuerdos fueron decididos mediante discusión abierta entre los jueces. Estos mismos jueces debieron evaluar también en qué medida aquellas propuestas clasificadas como analogías les resultaron novedosas en una escala Likert $(1=$ nada novedosa, $5=$ muy novedosa).

\section{Resultados y Discusión}

Los participantes generaron un total de 147 propuestas de analogías para los AM $(M=2,45$ de propuestas por participante por AM, $D E=0,70)$. Ciento veintinueve fueron clasificadas como analogías $(M$ $=2,15$ analogías por participante, $D E=0,94)$ y 18 fueron consideradas respuestas no analógicas. Estos niveles absolutos de propuestas de analogías resultan muy inferiores a los encontrados por Blanchette y Dunbar (2000), por lo que no puede descartarse que los altos niveles absolutos encontrados por estos autores se deban, en parte, a la recuperación de analogías conocidas con anterioridad al estudio. Por otro lado, la media obtenida por las analogías en cuanto a su carácter novedoso fue de 3,77 ( $D E=$ $0,84)$, lo que muestra claramente que los materiales elegidos fueron apropiados, en el sentido de no dar lugar a la recuperación de analogías culturalmente compartidas. Del conjunto total de analogías, el $73,6 \%$ fueron clasificadas como analogías interdominio y el $26,4 \%$ como intradominio. Al igual que en el experimento de Blanchette y Dunbar (2000), el porcentaje de analogías interdominio resultó superior al porcentaje de analogías intradominio, $\mathrm{Z}$ $=5,28, p=0,000$. Dado que estos resultados fueron obtenidos a partir de $10 \mathrm{AM}$ muy diversos en cuanto a los temas -ninguno, de acuerdo a nuestras estimaciones, de carácter público- y principios que ilustraban, el predominio relativo de propuestas interdominio obtenido por Blanchette y Dunbar (2000) no puede ser atribuido más que en parte al AM específico empleado y a la recuperación de analogías disponibles en la cultura. En resumen, aunque en términos absolutos la recuperación de análogos fue inferior a la obtenida por Blanchette y Dunbar (2000), los resultados de recuperación de analogías y el predominio de analogías interdominio siguen siendo muy altos en comparación a los obtenidos tradicionalmente por el paradigma de recepción, por lo que puede concluirse que el paradigma de producción logra altos niveles de recuperación y que promueve la recuperación de $\mathrm{AB}$ inter por sobre los $\mathrm{AB}$ intradominio, en contra de lo que ocurre en contextos experimentales.

\section{Experimento 2}

Nuestro segundo experimento tuvo como objetivo evaluar si, tal como postulan Blanchette y Dunbar (2000) y Dunbar (2001), una codificación estructural de $A B$, sumada a una tarea meta de generación de analogías, promueve altos niveles de recuperación de AB interdominio. Para ello se utilizó un procedimiento que combinó la tarea meta de generación de analogías con fines persuasivos (propia del paradigma de producción) con la provisión de $\mathrm{AB}$ de parte del experimentador (propia del paradigma de recepción). A diferencia de las consignas de codificación típicamente utilizadas en el paradigma de recepción -que según Blanchette y Dunbar (2000) promueven una codificación superficial- se emplearon consignas destinadas a estimular una codificación estructural de los AB.

\section{Método}

Participantes. Setenta estudiantes de segundo año de Psicología de la Universidad de Buenos Aires tomaron parte en el experimento de manera voluntaria. La muestra estuvo conformada por 51 mujeres y 19 varones, con edades entre 18 y 32 años $(M=21,20, D E=3,26)$.

Diseño y procedimiento. La variable distancia semántica entre los $\mathrm{AB}$ y los $\mathrm{AM}$ (dos niveles: intradominio e interdominio) recibió una manipulación intrasujeto. Los participantes pasaron por las dos fases del experimento (codificación y recuperación) de forma grupal, aunque trabajaron individualmente. La fase de codificación fue presentada oralmente como un experimento sobre comprensión lectora. Se les solicitó que leyeran atentamente las 10 historias que se les presentarían en un cuadernillo, prestando atención al principio o la idea abstracta que ilustraba cada una de ellas y a cómo lo ejemplificaba la historia, ya que más tarde deberían explicar algunas de estas historias a otro estudiante (esta consigna buscaba promover una codificación de los aspectos más abstractos y relevantes de los AB). Se les dijo además que, tras leer cada una, deberían responder dos preguntas que 
evaluaban la comprensión de lo leído. Se les aclaró que para ninguna de las dos tareas podrían volver a leer las historias. Estas consignas fueron también presentadas por escrito. Los participantes contaron con un máximo de 30 minutos para leer los $\mathrm{AB}$ y responder las preguntas. Tras la finalización de esta fase se les concedió un recreo de 20 minutos y se les pidió que volvieran para participar en un estudio sobre argumentación. Se les presentaron entonces dos tareas meta (extraídas del conjunto de AM del Experimento 1). Solo 2 de las 10 historias leídas por cada participante eran análogas a las dos historias meta (las 8 restantes eran de relleno): una constituía un $\mathrm{AB}$ interdominio de uno de los dos $\mathrm{AM}$ y la otra un $\mathrm{AB}$ intradominio del $\mathrm{AM}$ restante. Las consignas y los tiempos dados para estas tareas fueron idénticos a los del Experimento 1.

A los efectos de contabilizar como casos de recuperación todos aquellos $\mathrm{AB}$ que fueron efectivamente recordados -y no solo aquellos incluidos en las propuestas de analogías-, se diseñó un cuestionario destinado a detectar casos de $\mathrm{AB}$ recuperados pero no empleados. En la Tabla 1 puede observarse un esquema simplificado del procedimiento utilizado.

Tabla 1

Procedimiento del Experimento 2: Fases, Actividades y Objetivos

\begin{tabular}{|c|c|c|}
\hline Fase & Actividad & Objetivo \\
\hline \multirow{3}{*}{$\begin{array}{l}\text { Fase de codificación } \\
\text { (presentada como } \\
\text { experimento de } \\
\text { comprensión } \\
\text { lectora) } \\
30 \text { min. }\end{array}$} & $\begin{array}{l}\text { Este procedimiento se repite para el } \mathrm{AB} 1 \text {, el } \mathrm{AB} 2 \text { y las ocho } \\
\text { historias de relleno: }\end{array}$ & $\begin{array}{l}\text { Incorporación en memoria de largo plazo } \\
\text { (MLP) de los } \mathrm{AB} \text {. }\end{array}$ \\
\hline & 1. Lectura de una historia. & $\begin{array}{l}\text { Verificar que los alumnos realicen una } \\
\text { lectura atenta de los } \mathrm{AB} \text {. }\end{array}$ \\
\hline & \multicolumn{2}{|l|}{ 2. Respuesta a dos preguntas de comprensión. } \\
\hline $\begin{array}{l}\text { Recreo } \\
20 \text { min. }\end{array}$ & $\begin{array}{l}\text { Los alumnos se retiran del aula, realizan sus actividades y } \\
\text { retornan al aula. }\end{array}$ & $\begin{array}{l}\text { Reforzar la separación contextual entre la } \\
\text { fase de codificación y la fase de recupe- } \\
\text { ración. }\end{array}$ \\
\hline \multirow{11}{*}{$\begin{array}{l}\text { Fase de recupera- } \\
\text { ción } \\
\text { (presentada como } \\
\text { experimento de } \\
\text { argumentación) } \\
\quad 44 \text { min. }\end{array}$} & \multirow{5}{*}{$\begin{array}{l}\text { 1. Lectura del AM } 1 \text { (intradominio con respecto a AB 1). } \\
\text { 2. Tarea de generación de analogías para el AM } 1 . \\
\text { 3. Lectura del AM } 2 \text { (interdominio con respecto a AB 2). } \\
\text { 4. Tarea de generación de analogías para el AM } 2 . \\
\text { 5. Cuestionario, parte } 1 \text { (se repite de la misma forma para el } \\
\text { AM } 1 \text { y el AM 2) }\end{array}$} & \multirow[t]{5}{*}{ Registrar analogías propuestas. } \\
\hline & & \\
\hline & & \\
\hline & & \\
\hline & & \\
\hline & $\begin{array}{l}\text { a) Se presenta una breve descripción del AM } 1 . \\
\text { b) Se pregunta si hubo recuperación de alguna/s }\end{array}$ & $\begin{array}{l}\text { Registrar recuperaciones de } \mathrm{AB} \text { no inclui- } \\
\text { das en las analogías propuestas. }\end{array}$ \\
\hline & $\begin{array}{l}\text { historias de la fase de codificación que no resulta- } \\
\text { ron incluidas en las propuestas de analogías (si la } \\
\text { respuesta es si se pasa a "c" y luego a la parte } 2 \text {; si } \\
\text { es no se pasa a la parte 2). }\end{array}$ & \\
\hline & $\begin{array}{l}\text { c) Se presentan referencias superficiales a las diez his- } \\
\text { torias base y el participante marca cuál/es recordó. }\end{array}$ & \\
\hline & $\begin{array}{l}\text { 6. Cuestionario, parte } 2 \text { (se repite de la misma forma para el } \\
\mathrm{AB} 1 \text { y el } \mathrm{AB} 2 \text { ) }\end{array}$ & \\
\hline & $\begin{array}{l}\text { a) Se presenta una referencia al AB cuya disponibilidad } \\
\text { se intentaba evaluar. }\end{array}$ & \\
\hline & $\begin{array}{l}\text { b) Se pide al participante que narre dicha historia con el } \\
\text { máximo nivel posible de detalle. }\end{array}$ & $\begin{array}{l}\text { Determinar si el AB cuya recuperación se } \\
\text { intenta evaluar se encontraba disponible } \\
\text { en MLP. }\end{array}$ \\
\hline
\end{tabular}

Nota. Para dar como recuperado un AB este debe: a) haber sido incluido en las propuestas de analogías, o b) haber sido informado como recuperado en la parte 1 del cuestionario. En ambos casos su contenido debe haber sido además descrito correctamente, algo que evaluaron jueces independientes. Para dar como no recuperado un $\mathrm{AB}$ el participante debió haber sido capaz de describirlo en la parte 2 del cuestionario. 
Materiales. Se utilizaron 10 conjuntos de tres historias cada uno, conformados por un AM de final abierto (extraído del Experimento 1) y dos $\mathrm{AB}$ con final cerrado. Para cada AM se derivó un $A B$ intradominio y un $\mathrm{AB}$ interdominio de entre $220 \mathrm{y}$ 240 palabras. Ambos AB se encontraban resueltos a favor de una misma línea de acción, por lo que la misma forma de posicionarse frente al AM podía ser derivada, por analogía, desde cualquiera de los dos AB. Por ejemplo, para el AM ya referido que tenía como protagonista a un investigador que debía decidir si conservar unas muestras de un virus, los $\mathrm{AB}$ sugerían su total eliminación. Mientras el $\mathrm{AB}$ intradominio hacía referencia a las desastrosas consecuencias provocadas por la desaparición de una probeta con muestras de aftosa de un centro de investigaciones, el $\mathrm{AB}$ interdominio hacía referencia a las graves consecuencias que produjo que dos oficiales del ejercito ruso vendieran ojivas nucleares a países de medio oriente (en el Anexo 2 se incluyen los AB derivados a partir de uno de los dos AM presentados en el Anexo 1). Los AB estaban incluidos en un cuadernillo, en cuya primera hoja aparecían las consignas ya dadas oralmente. Intercaladas entre una historia y la siguiente aparecían dos preguntas con formato de respuesta de verdadero o falso, las que fueron utilizadas para descartar del análisis de datos a aquellos participantes que dieran muestras de no haber realizado una lectura atenta de las historias. Se confeccionaron dos versiones diferentes de los cuadernillos. La versión 1 contenía $5 \mathrm{AB}$ intradominio y $5 \mathrm{AB}$ interdominio, todos ellos pertenecientes a conjuntos diferentes. La versión 2 recogía los $10 \mathrm{AB}$ complementarios. Los materiales de las tareas meta fueron idénticos a los empleados en el Experimento 1. El orden de presentación de los AB y los AM fue contrabalanceado. A efectos de asegurarnos que los $A B$ intra e interdomino relativos a un AM mantenían con este un mismo parecido estructural, condujimos un estudio complementario en el que apareamos cada uno de los $10 \mathrm{AM}$ con uno de sus correspondientes $\mathrm{AB}, \mathrm{y}$ pedimos a una muestra independiente de 30 estudiantes de segundo año de Psicología de la Universidad de Buenos Aires que juzgaran, mediante una escala tipo Likert de 1 a 7 , en qué medida consideraban que ambas historias constituían una buena analogía. Ningún participante tuvo que evaluar dos AB tomados de un mismo conjunto. Todas las analogías presentadas obtuvieron puntuaciones promedio mayores a 5. Las analogías intradominio $(M=6,06, D E=0,77)$ y las analogías interdominio
$(M=5,90, D E=0,75)$ no difirieron en cuanto a su parecido estructural, $t=1,90, p=0,059$.

La primera parte del cuestionario escrito, destinado a detectar recuperaciones de $\mathrm{AB}$ no incluidos en las analogías propuestas, presentaba un resumen del primer AM y pedía luego al participante que informara, marcando sí o no, si durante la realización de la tarea de generación vinculada a dicho AM había recordado, aunque fuera de manera fugaz, alguna de las narraciones leídas durante la experiencia anterior sobre comprensión lectora, que no hubiera utilizado en sus propuestas de analogías. En el caso de haber contestado afirmativamente, debían consignar las historias recordadas. Para ello se les presentaban referencias de menos de 10 palabras de cada uno de los AB leídos por ese participante (e.g, "la historia de las ojivas nucleares") y se les pedía que marcasen aquella (o aquellas) que en su momento habían sido recordadas. Este procedimiento se repetía para el AM restante. Dado que no es adecuado computar como éxito o fracaso de recuperación analógica los casos en los que los $\mathrm{AB}$ no se encontraban debidamente almacenados en la memoria, la segunda parte del cuestionario buscaba evaluar la disponibilidad en la memoria de los dos $\mathrm{AB}$ relevantes recibidos por cada participante. A tal efecto, se les presentó una breve referencia de los $\mathrm{AB}$ cuya disponibilidad se buscaba evaluar y se les pidió que reprodujeran dichas historias con el máximo detalle posible. Esta tarea estaba destinada a excluir del análisis de datos los ensayos en los que los participantes no dieran muestras de disponer del AB en la memoria. Dos jueces independientes evaluaron, utilizando en una escala de 0 a 3 , la calidad de la recuperación $(0=$ ningún recuerdo, 1 = hechos aislados, pero sin episodio central, 2 = episodio central, 3 = episodio central más detalles). Se dio por válido un recuerdo cuando ambos evaluadores otorgaron un puntaje igual o superior a 2. El análisis de datos excluyó los casos en los que los participantes no lograron recordar los $\mathrm{AB}$ de manera satisfactoria durante el cuestionario. Utilizando este mismo criterio de evaluación, los jueces determinaron además la calidad del recuerdo de los $\mathrm{AB}$ experimentales incluidos en las propuestas de analogías de los participantes.

\section{Resultados y Discusión}

Dos nuevos jueces fueron capacitados para analizar las propuestas de analogías de los participantes, de la misma forma que en el Experimento 1. El acuerdo entre los jueces alcanzó el $88 \%$, respecto 
al carácter analógico-no analógico de las propuestas de analogías (Kappa $=0,58, p<0,001$ ) y el $77 \%$ respecto al carácter inter o intradominio de las resupestas analógicas (Kappa $=0,48, p<0,001)$. Los casos en los que no hubo acuerdo fueron resueltos por discusión abierta entre los jueces. Excluyendo las respuestas basadas en la recuperación de $\mathrm{AB}$ experimentales, los participantes realizaron un total de 307 propuestas de analogías, lo que representa una media de $2,19(D E=0,88)$ propuestas por tarea meta por participante. De estas propuestas, 268 fueron clasificadas como analogías $(M=1,91$ analogías por participante, $D E=0,92$ ) y 39 fueron consideradas respuestas no analógicas.

Al igual que en el Experimento 1, pudo verificarse que los niveles absolutos de recuperación de $\mathrm{AB}$ fueron muy inferiores a los reportados por Blanchette y Dunbar (2000). Del conjunto total de analogías, el 71,27\% fueron clasificadas como analogías interdominio y el $28,73 \%$ como intradominio, proporción que sigue un patrón muy similar al obtenido por Blanchette y Dunbar (2000) y en nuestro primer experimento.

En este segundo experimento nuestro interés se centró, empero, en la recuperación de $\mathrm{AB}$ experimentales. Todos los participantes respondieron correctamente más del $80 \%$ de las preguntas que seguían a la lectura de cada $\mathrm{AB}$, por lo que ningún participante fue excluido del análisis de datos. De los 140 casos potenciales de recuperación de AB, la mitad eran de recuperación de $\mathrm{AB}$ intradominio y la otra mitad de AB interdominio. Fueron contabilizados como casos de recuperación de $\mathrm{AB}$ aquellos en los que los participantes: 1) usaron el $A B$ en sus propuestas de analogías, siendo su recuerdo evaluado como aceptable por los jueces, o 2) reconocieron haber pensado en dicho $\mathrm{AB}$ durante la generación de analogías (primera parte del cuestionario), dando además muestras de poder recuperarlo (segunda parte del cuestionario). El análisis de las respuestas a la segunda parte del cuestionario determinó que 10 $\mathrm{AB}$ intradominio y $13 \mathrm{AB}$ interdominio no estaban disponibles en la memoria de los participantes, por lo que dichos ensayos fueron excluidos de todo análisis. En promedio, los $\mathrm{AB}$ fueron recuperados en el $43 \%$ de los ensayos. Los AB intradominio fueron recuperados en el $60 \%$ de los ensayos, mientras que los $\mathrm{AB}$ interdominio fueron recuperados en el $26 \%$ de los ensayos, lo que muestra un efecto negativo de la distancia semántica entre $\mathrm{AB}$ y AM sobre la probabilidad de recuperar un $\mathrm{AB}$ determinado desde memoria, $\chi^{2}(1, N=117)=13,49, p=0,000$. Del total de AB recuperados, el 29\% fueron finalmente empleados en las analogías propuestas por los participantes. Los $\mathrm{AB}$ interdominio recuperados difirieron de los AB intradominio en cuanto a la probabilidad de resultar utilizados en las propuestas de analogías persuasivas, $\chi^{2}(1, N=51)=9,58, p=0,002$. Mientras que solo un $17 \%$ de los $\mathrm{AB}$ intradominio recuperados resultaron finalmente empleados, el $60 \%$ de los $\mathrm{AB}$ interdominio recuperados resultaron utilizados en las propuestas de analogías.

Los resultados obtenidos no apoyan la hipótesis postulada por Blanchette y Dunbar (2000) y Dunbar (2001) que una codificación estructural de AB sumada a una tarea meta de generación de analogías resulta suficiente para promover altos niveles absolutos y relativos de recuperación de $\mathrm{AB}$ interdominio. Por el contrario, parecen consistentes con los típicamente obtenidos en estudios sobre solución de problemas (e.g., Catrambone \& Holyoak, 1989; Gentner, Lowenstein \& Thompson, 2004; Gick \& Holyoak, 1980, 1983; Spencer \& Weisberg, 1986), los que han demostrado que la codificación estructural de un solo $\mathrm{AB}$ combinada con una tarea que promueve una atención a aspectos estructurales del AM (una tarea de solución de problemas parece propicia para este fin) no resultan suficientes para provocar altos niveles de recuperación interdominio, requiriéndose la comparación sistemática entre dos o más $\mathrm{AB}$ para que se produzca dicha transferencia.

\section{Discusión General}

Una larga tradición de estudios experimentales que han empleado el paradigma de recepción ha demostrado que la recuperación de análogos interdominio es muy reducida, tanto en términos absolutos como en relación a la recuperación de análogos intradominio. Utilizando un paradigma de producción, Blanchette y Dunbar (2000, Experimento 2) encontraron un patrón opuesto de resultados. Dado que el tema meta utilizado por estos autores había tenido gran cobertura mediática en los meses previos al estudio, la diferencia encontrada respecto a los resultados del paradigma de recepción podría atribuirse a que sus participantes utilizaron analogías preestablecidas respecto al AM, ya sea escuchadas en los medios o disponibles en la cultura. En nuestro primer experimento buscamos evidencias capaces de hacer que los resultados de Blanchette y Dunbar (2000) fueran menos vulnerables a esta objeción. Para reducir la probabilidad que nuestros participantes propusieran analogías conocidas previamente en 
vez de crearlas, empleamos 10 AM que trataban muy diversos temas, ninguno de carácter público.

Nuestros resultados mostraron una recuperación de análogos muy inferior a la encontrada por Blanchette y Dunbar (2000), aunque superior a la normalmente obtenida en estudios que emplean el paradigma de recepción. Más importante aún, esta recuperación resultó predominantemente interdominio. Dado que se utilizaron 10 AM diferentes -ninguno de carácter público-, el predominio de recuperaciones interdominio obtenido por Blanchette y Dunbar no puede ser atribuido al carácter público de la temática usada por estos autores como AM.

Blanchette y Dunbar (2000) y Dunbar (2001) propusieron una posible explicación de por qué el paradigma de producción, a diferencia del paradigma de recepción, induce altos niveles de recuperación de $\mathrm{AB}$ interdominio. Por una parte, los $\mathrm{AB}$ recogidos fuera del laboratorio serían codificados en términos de sus aspectos estructurales, a diferencia de los $\mathrm{AB}$ experimentales, cuyas consignas de lectura no promoverían una codificación estructural. Por otra parte, la tarea de generación de analogías dirige la atención a aspectos estructurales del AM, mientras que las tareas meta de recuerdo del paradigma de recepción no lo hacen. En el Experimento 2 de este estudio pusimos a prueba la hipótesis que una codificación estructural del AB combinada con una tarea de generación de analogías da lugar a altas tasas absolutas y relativas de recuperación interdominio. Para ello empleamos un paradigma "mixto", en el que los $\mathrm{AB}$ fueron provistos por el experimentador (algo propio del paradigma de recepción) y una tarea de generación de analogías (algo propio del paradigma de producción). Para la lectura de los $\mathrm{AB}$ se dio una consigna destinada a promover un procesamiento estructural de los mismos. De encontrar una alta recuperación interdominio, razonamos, contaríamos con una prueba indirecta a favor de la hipótesis ofrecida por estos autores para explicar la eficacia del paradigma de producción. Los resultados obtenidos fueron, sin embargo, los típicamente obtenidos con el paradigma de recepción: una alta recuperación intradominio y una baja recuperación interdominio. Los datos mostraron que una codificación estructural del AM y una tarea de generación de analogías no alcanzan para replicar el patrón de resultados del paradigma de producción, por lo que esta explicación sobre lo que ocurre con el paradigma de producción no parece suficiente. No puede descartarse, sin embargo, que nuestra consigna de procesamiento de los $\mathrm{AB}$ no haya sido suficiente para promover una codificación estructural de estos, algo que podríamos poner a prueba mejorando dichas consignas en futuros estudios. Una interpretación alternativa de los datos encontrados podría consistir en postular que los $\mathrm{AB}$ recogidos fuera del laboratorio guardan alguna otra particularidad que determina que se encuentren más accesibles que los $\mathrm{AB}$ de laboratorio.

Los resultados obtenidos por Blanchette y Dunbar (2000, Experimento 2) y por nosotros en los dos experimentos de este estudio constituyen un desafío tanto para una larga tradición empírica sobre recuperación analógica, según la cual las claves superficiales dominan la recuperación de $\mathrm{AB}$ (e.g., Catrambone, 2002; Gentner et al., 1993; Wharton et al., 1994), como para los principales modelos computacionales sobre recuperación analógica los que, al simular la recuperación humana, otorgan un peso preeminente a las semejanzas superficiales en la recuperación (e.g., Forbus, Gentner \& Law, 1995; Thagard, Holyoak, Nelson \& Gochfeld, 1990; Hummel \& Holyoak, 1997).

Un aspecto que sería importante indagar en futuras investigaciones se refiere a diferenciar, dentro del conjunto de $\mathrm{AB}$ propuestos para un $\mathrm{AM}$ en una tarea de generación, entre $\mathrm{AB}$ recuperados de la memoria y AB inventados de forma ad hoc para el AM (De la Fuente \& Minervino, 2004). Otro aspecto que amerita atención con respecto al paradigma de producción se refiere a indagar cuántos $\mathrm{AB}$ resultan recuperados pero no incluidos en las analogías propuestas por los participantes (Blanchette \& Dunbar, 2000). Mientras que la estimación de ambos fenómenos puede implementarse mediante un paradigma "mixto" como el empleado en nuestro Experimento 2, no parece sencillo realizarla mediante un paradigma de producción. Aunque los resultados de nuestro segundo experimento solo informan del tratamiento que reciben los $\mathrm{AB}$ presentados por los experimentadores, la comparación entre los patrones de recuperación de $\mathrm{AB}$ y los patrones de $\mathrm{AB}$ finalmente empleados muestra evidencia de fuertes procesos de selección de los análogos recuperados. Por razones no del todo conocidas, las probabilidades de emplear un $\mathrm{AB}$ que ha sido recuperado resultaron más de tres veces superiores cuando dicho $A B$ es interdominio que cuando se trata de un $\mathrm{AB}$ intradominio. Estos datos sugieren que algo similar podría tener lugar en el paradigma de producción. En resumen, sería interesante que futuras investigaciones logren determinar cuánto hay de recuperación y de qué tipo en 
el paradigma de producción. Estos controles podrían permitir conocer mejor los niveles absolutos y relativos que efectivamente tienen lugar en el paradigma de producción y avanzar hacia una explicación de por qué ocurren.

\section{Referencias}

Blanchette, I. \& Dunbar, K. (1997). Constraints underlying analogy use in a real world context: Politics. En M. G. Shafto \& P. Langley (Eds.), Proceedings of the Nineteenth Annual Conference of the Cognitive Science Society (p. 867). Mahwah, NJ: Lawrence Erlbaum.

Blanchette, I. \& Dunbar, K. (2000). How analogies are generated: The roles of structural and superficial similarity. Memory \& Cognition, 28, 108-124.

Catrambone, R. (2002). The effects of surface and structural feature matches on the access of story analogs. Journal of Experimental Psychology: Learning, Memory, and Cognition, 28, 318-334.

Catrambone, R. \& Holyoak, K. J. (1989). Overcoming contextual limitations on problem-solving transfer. Journal of Experimental Psychology: Learning, Memory, and Cognition, 15, 1147-1156.

De la Fuente, J. \& Minervino, R. A. (2004). Pensamiento analógico. En M. Carretero \& M. Asensio (Coord.), Psicología del pensamiento (pp. 193-214). Madrid: Alianza.

Dunbar, K. (2001). The analogical paradox: Why analogy is so easy in naturalistic settings, yet so difficult in the psychology laboratory? En D. Gentner, K. J. Holyoak \& B. K. Kokinov (Eds.), The analogical mind: Perspectives from cognitive science (pp. 313-334). Cambridge, MA: MIT Press.

Forbus, K. D., Gentner, D. \& Law, K. (1995). MAC/FAC: A model of similarity-based retrieval. Cognitive Science: A Multidisciplinary Journal, 19, 141-205.

Gentner, D. (1983). Structure-mapping: A theoretical framework for analogy. Cognitive Science: A Multidisciplinary Journal, 7, 155-170.

Gentner, D. (1989). The mechanisms of analogical transfer. En S. Vosniadou \& A. Ortony (Eds.), Similarity and analogical reasoning (pp. 199-242). Cambridge, Inglaterra: Cambridge University Press.

Gentner, D., Holyoak, K. J. \& Kokinov, B. N. (Eds.) (2001). The analogical mind: Perspectives from cognitive science. Cambridge, MA: MIT Press.

Gentner, D., Loewenstein, J. \& Thompson, L. (2004). Analogical encoding: Facilitating knowledge transfer and integration. En K. Forbus, D. Gentner \& T. Regier (Eds.), Proceedings of the $26^{\text {th }}$ Annual Conference of the Cognitive Science Society (pp. 450-455). Mahwah, NJ: Lawrence Erlbaum.

Gentner, D., Rattermann, M. J. \& Forbus, K. D. (1993). The roles of similarity in transfer: Separating retrievability from inferential soundness. Cognitive Psychology, 25, 431-467.

Gick, M. L. \& Holyoak, K. J. (1980). Analogical problem solving. Cognitive Psychology, 12, 306-355.

Gick, M. L. \& Holyoak, K. J. (1983). Schema induction and analogical transfer. Cognitive Psychology, 12, 306-355.

Holyoak, K. J. \& Thagard, P. R. (1995). Mental leaps: Analogy in creative thought. Cambridge, MA: MIT Press.

Hummel, J. E. \& Holyoak, K. J. (1997). Distributed representations of structure: A theory of analogical access and mapping. Psychological Review, 104, 427-466.

Lakoff, G. \& Turner, M. (1997). More than cool reason: A field guide to poetic metaphor. Chicago: University of Chicago Press.

Minervino, R. A. (2007). La capacidad de la teoría de proyección de la estructura y la teoría de la metáfora conceptual para explicar la generación y el uso de metáforas conceptuales: una comparación crítica. Subjetividad y Procesos Cognitivos, 1, 28-41.

Minervino, R. A. \& Oberholzer, N. (2007). Falsa memoria de inferencias analógicas y cambio representacional. Anuario de Psicología, 38, 129-146.

Rattermann, M. J. \& Gentner, D. (1987). Analogy and similarity: Determinants of accessibility and inferential soundness. En E. E. Smith (Ed.), Proceedings of the Ninth Annual Conference of the Cognitive Science Society (pp. 23-35). Hillsdale, NJ: Lawrence Erlbaum.

Spencer, R. M. \& Weisberg, R. W. (1986). Context-dependent effects on analogical transfer. Memory \& Cognition, 14, 442-449.

Thagard, P., Holyoak, K. J., Nelson, G. \& Gochfeld, D. (1990). Analog retrieval by constraint satisfaction. Artificial Intelligence, 46, 259-310.

Wharton, Ch. M., Holyoak, K. J., Downing, P. E., Lange, T. E., Wickens, T. D. \& Melz, E. R. (1994). Below the surface: Analogical similarity and retrieval competition in reminding. Cognitive Psychology, 26, 64-101.

Wharton, Ch. M., Holyoak, K. J. \& Lange, T. E. (1996). Remote analogical reminding. Memory \& Cognition, 24, 629-643.

\section{Anexo 1}

Ejemplos de Tareas Meta, Experimentos 1 y 2

\section{Ejemplo 1}

La Avenida Colón era muy empinada, por lo que, aunque no estaba prohibido, se recomendaba no estacionar el auto. Si alguien quería hacerlo, debía poner el freno de mano. El hermano de Mauro siempre le decía que era un peligro dejar estacionado el coche allí, pero Mauro no le hacía caso. Hasta que un día ocurrió lo que podía ocurrir. El auto había quedado estacionado en dirección hacia abajo y sin ningún otro auto por delante de él. Mauro había olvidado ponerle el freno de mano. Fue así que el auto comenzó a deslizarse lentamente hasta llegar a una velocidad de $20 \mathrm{~km}$./h. Dos niñas cruzaban la calle; una de ellas logró esquivar el auto, pero la otra resultó atropellada, aunque los daños físicos que recibió fueron leves. Mauro estaba en su oficina cuando tuvo lugar el episodio. Cuando lo llamaron acudió al lugar de forma urgente y muy preocupado.

Suponga que usted es el abogado de la niña atropellada. Quiere convencer a Mauro, haciendo uso de analogías, que él es el responsable del accidente y de que perderá el juicio, ya que el dueño de un auto debe hacerse cargo de las cosas que pasen con él, más allá de su participación directa o no en lo ocurrido. Escriba a continuación todas las analogías 
que podría emplear. No se preocupe tanto por si las analogías son buenas o no; queremos saber qué analogías se le han ocurrido.

\section{Ejemplo 2}

Juan trabajaba desde chico en el bodegón de su padre. Hacía años que tenían los mismos empleados, que aunque no eran demasiado profesionales, resultaban confiables y relativamente accesibles. Cuando Juan se hizo cargo del negocio, el barrio había mejorado mucho, con lo que se vio forzado a imprimirle al restaurante una cierta renovación. El cambio más agresivo fue reemplazar al antiguo cocinero por un chef con formación en gastronomía internacional. El menú ganó en sofisticación, pero aparecieron dificultades. Algunas novedades tuvieron éxito, pero otras no convencieron a la clientela tradicional. Por otro lado, el chef se resistía a seguir elaborando los platos clásicos del local. Juan no pudo con estas dificultades y al poco tiempo reemplazó al chef. Pero la situación no mejoró. Así, desde hace algunos años, viene probando distintos chefs sin encontrar una solución satisfactoria. Los clientes se quejan por tantos cambios y Juan reacciona reemplazando al cocinero. En el presente la afluencia de gente es especialmente escasa y Juan está al borde de realizar un nuevo cambio.

Suponga que usted es cliente y amigo de Juan, y que quiere convencerlo que lo que más le conviene es seguir durante un tiempo más con el chef actual. Escriba a continuación todas las analogías que podría emplear. No se preocupe tanto por si las analogías son buenas o no; queremos saber qué analogías se le han ocurrido.

\section{Anexo 2}

Ejemplo de Dos AB Empleados para un AM, Experimento 2 (Corresponden al AM Presentado como Ejemplo 2 en el Apéndice A)

\section{$A B$ interdominio}

Pedro empezó a jugar al golf a los once años, con unos viejos palos heredados de su abuelo. Estos palos habían perdido actualidad pero Pedro se entendía bien con ellos. Por otra parte, los palos de golf eran artículos importados y en épocas de estudiante no tenía dinero para comprar unos nuevos. Después de recibido, a instancias de sus amigos, juntó varios sueldos y compró una bolsa nueva. Sin embargo, su juego no mejoró; más bien lo contrario: en promedio pegaba más lejos que antes pero de manera muy irregular. A veces pegaba fuertísimo y se pasaba de la bandera, otras se quedaba corto. A los tres meses, harto de estas fluctuaciones, decidió pasarse a otro modelo, pero sin éxito. Así, a lo largo de cuatro años alternó marcas y modelos, con un costo muy alto en dinero y frustraciones. Leía obsesivamente las revistas de golf y ensayaba una técnica tras otra para mejorar el golpe. Cuando se casó, el esfuerzo económico para mantener el departamento lo obligó a suspender la rutina de renovar los palos. El resultado fue mágico: poco a poco se fue acostumbrando a sus palos actuales. Recuperó la confianza perdida y al poco tiempo volvió a ponerse al nivel de sus amigos. Se dio cuenta que no era que los anteriores hayan sido peores sino que lo que hacía falta era concederles un mínimo de tiempo para "agarrarles la mano".

\section{$A B$ intradominio}

Mariana fue criada desde recién nacida por una niñera muy buena, que siguió vinculada a la familia hasta el nacimiento del menor de sus hermanos. Cuando Mariana tuvo a su primer hijo consiguió que la asistiera su vieja niñera. La señora tenía sus achaques, pero cobraba poco y se entendía muy bien con Mariana. Cuando tuvo al segundo, ahorró para contratar una niñera profesional. Sin embargo, el cambio no le simplificó las cosas, más bien lo contrario. La nueva chica estaba preparada para suplir a la madre en más tareas, pero tenía sus aristas conflictivas: algunas veces seguía al pie las instrucciones, pero otras hacía caso omiso de las indicaciones de la madre. A los tres meses, harta de estas fluctuaciones, cambió de niñera. Pero esta historia se repitió muchas veces: no conseguía entenderse con ellas y al poco tiempo las despedía, afrontando un alto costo en dinero y frustraciones. Cuando tuvo a su cuarto hijo las exigencias económicas la forzaron a centrarse en su trabajo, dejando de obsesionarse por la crianza de los chicos y suspendiendo el cambio de niñeras. Mágicamente, esto tuvo un efecto positivo: comenzó a entenderse cada vez mejor con su actual niñera y al poco tiempo logró delegar en ella tanto o más cosas que con la primera. Se dio cuenta que no era que las anteriores hayan sido malas sino que en todos los casos se requiere un cierto tiempo para llegar a entenderse bien. 